\title{
Is candy eating a way to control body weight?
}

\author{
Mikael Fogelholm and Inge Tetens
}

T he increasing prevalence of overweight and obesity has called for actions already in place for years. Unfortunately, the results so far have been mostly disappointing. The role of science in combating obesity is to provide evidence for decision-making at political (national and local) level, in food industry, in health care settings and in educational planning. Evidently, more data are needed to understand the detailed aetiology of obesity and how to combat obesity at population and individual levels.

In addition to physical activity, diet is a major factor affecting energy balance and subsequently weight change. Most of the studies looking at the impact of diet on weight change have concentrated on the proportions of macronutrients (as percent in total energy intake, $\mathrm{E} \%$ ) or on consumption of specific foods.

Recently, several systematic reviews and meta-analyses have addressed the role of carbohydrates in obesity. Cross-sectional studies tend to show an inverse relation between the proportion of dietary carbohydrates $(\mathrm{E} \%)$ and obesity (1). In contrast, cohort studies and randomised intervention trials do not provide strong evidence that increasing or decreasing the proportion of dietary carbohydrates has a significant independent effect on body weight maintenance (1).

Sugar-rich foods are usually low in dietary fibre and at least moderately high in energy density. In theory, both properties could link high sugar intake with obesity. In an extensive narrative review, van Baak and Astrup (2) concluded that there is insufficient evidence that an exchange of sugar to nonsugar (starch- and/or fibrerich) carbohydrates would assist in body weight reduction. Very few population studies report on the association between sugar-rich food intake and weight gain. In a study using the EPIC cohort in Potsdam, Schulz et al. (3) reported that intake of high sugar foods, i.e. sweets, was significantly predictive of large weight gain. However, there is stronger epidemiological $(1,2)$ and less consistent evidence from randomised intervention trials (4) that sugar-sweetened beverages may induce obesity.

Since the data on the role of carbohydrate on the development of obesity are still inconclusive, more research on this topic is warranted. Therefore, studies like the one published in Food and Nutrition Research on the association of candy consumption on body weight (5) might give some new insights into this important issue.
O'Neil et al. (5) have had access to a large data set, a total of 7,049 children and 4,132 adolescents from the National Health and Nutrition Examination Survey (NHANES). By using one 24-hour dietary recall, they divided the participants into two groups: candy consumers and nonconsumers. Energy intake was higher in candy consumers, but after adjusting for gender, ethnicity, age and energy intake, the consumers' mean weight was significantly lower by $1.4 \mathrm{~kg}$. The authors concluded that candy consumers were less likely to be overweight than nonconsumers.

The large number of participants in the study of O'Neil et al. (5) is a definite strength, and the authors should be praised for carrying out their research. However, one needs to be somewhat careful with the interpretation and practical implications of their main finding. There are several methodological and even philosophical reasons for this request.

First, the authors used one 24-hour recall to dichotomise participants into consumers and nonconsumers. Although a 24-hour recall may very well indicate the mean intake of group level, day-to-day variations in food intake decrease the reliability on individual level (6). It is likely that both 'heavy-users' and 'never-users' of candy are classified correctly by the 24-hour recall. In contrast, the extent of misclassification among all other participants can only be guessed. Misclassifications tend to preclude from finding significant associations, that is, they increase the likelihood for type II errors.

Another methodological concern is related to the adjustments. Since changes in weight should reflect energy balance, energy intake must be considered a major factor. This leads to the question, whether adjustment for energy intake is warranted? The concern on adjusting for energy intake is emphasised by looking at the results: according to data with adjusting only for gender, ethnicity and age, energy intake was significantly higher in candy consumers. The difference in mean intake (9.4 vs. 8.3 MJ/day) was not only statistically significant: if a difference of this magnitude is real, it ought to be associated with energy balance. However, when the outcome was changed to weight, and energy intake was included as covariate, the weight difference between consumers and nonconsumers was favouring consumers.

The third concern is more philosophical: a majority of studies assessing the relationships between diet and 
health are using a reductionistic approach (7): the whole diet is split into parts, namely, macronutrients, subclasses of macronutrients (like different carbohydrates or fatty acids), food groups and individual foods. While this is understandable and even necessary in many cases, the critical question still is whether the reality can be reconstructed by its parts (7). Indeed, we nutritionists often say that the whole diet is much more than the sum of the parts.

Studying the association between sugar and body weight is indeed a very reductionistic approach. Carbohydrates are a part of the whole diet. Sugars are a part of total carbohydrates and solid sugars a part of total sugars. The sugar from candy is eventually a part of all solid sugars. O'Neil et al. (5) found that the contribution of candy to total added sugars was very little. The question then is whether variation in only this single component could even in theory have any significant real effects on body weight? And if an association of effect between candy and body weight is found, would it tell more about the overall dietary habits or even dietary patterns and other health behaviours of candy consumers (or nonconsumers) than about the effects of candy per se?

A final comment is related to the design. The study is cross-sectional and can therefore not give any insight on causality. An explanation for the surprising finding could be that children and adolescents with obesity have decreased their intake of candy. This would mean that reported candy consumption in this study reflects consequences of obesity, not causes. Therefore, the level of evidence gained from cross-sectional studies may not be strong enough to be used in decision-making. Crosssectional designs are not worthless, though, since they create hypotheses to be tested in prospective settings and preferably in randomised intervention trials.

What would the implications of the finding by O'Neil et al. (5) on scientific research be? Would it point towards the need of an intervention where the hypothesis is that increased intake of candy prevents body weight gain or reduces body weight in overweight participants? It is doubtful that any ethical committee would be happy about this kind of a proposal.

\section{References}

1. van Dam RM, Seidell JC. Carbohydrate intake and obesity. Eur J Clin Nutr 2007; 61: S75-99.

2. van Baak MA, Astrup A. Consumption of sugars and body weight. Obes Rev 2009; 10: 9-23.

3. Schulz M, Kroke A, Liese AD, Hoffmann K, Bergmann MM, Boeing H. Food groups as predictors for short-term weight changes in men and women of the EPIC-Potsdam cohort. J Nutr 2002; 132: $1335-40$

4. Mattes RD, Shikany JM, Kaiser KA, Allison DB. Nutritively sweetened beverage consumption and body weight: a systematic review and meta-analysis of randomized experiments. Obes Rev 2011; 12: 346-65.

5. O'Neil CE, Fulgoni VL, Nicklas TA. Association of candy consumption with body weight measures, other health risk factors for cardiovascular disease, and diet quality in US children and adolescents: NHANES 1999-2004. Food Nutr Res 2011; 55: 5794. DOI: 10.3402/fnr.v55i0.5794.

6. Gibson RS. Principles of nutritional assessment. 2nd ed. Oxford: Oxford University Press; 2005. p. 1-908.

7. Hoffman I. Transcending reductionism in nutrition research. Am J Clin Nutr 2003; 78: 514S-6S.

Mikael Fogelholm, Professor in Nutrition Department of Food and Environmental Sciences University of Helsinki, Finland Email: mikael.fogelholm@helsinki.fi Inge Tetens, Professor National Food Institute, Division of Nutrition Technical University of Denmark Søborg Denmark Email: intet@food.dtu.dk 